\title{
US National Academies report on the Frontiers of Materials Research
}

$\mathrm{M}$ aterials research is vital across many important societal sectorsfrom health and wellness, to energy and sustainability, to national security. It is also constantly evolving, with advancements in technology, integration of computational methods, and the development of new means of materials synthesis, processing, and characterization. "Because of the depth and breadth of materials science [and engineering], it is important to periodically look at the applications across the landscape of different disciplines and technologies - to highlight the work that's been done as well as the directions for new discovery-and to make this information available to both scientists and policymakers," says Damon Dozier, director of government affairs for the Materials Research Society.

This is a task that was recently taken on by the US National Academies of Sciences, Engineering, and Medicine (Academies) at the behest of the National Science Foundation (NSF) and the Department of Energy (DOE). The resulting study, entitled Frontiers of Materials Research:

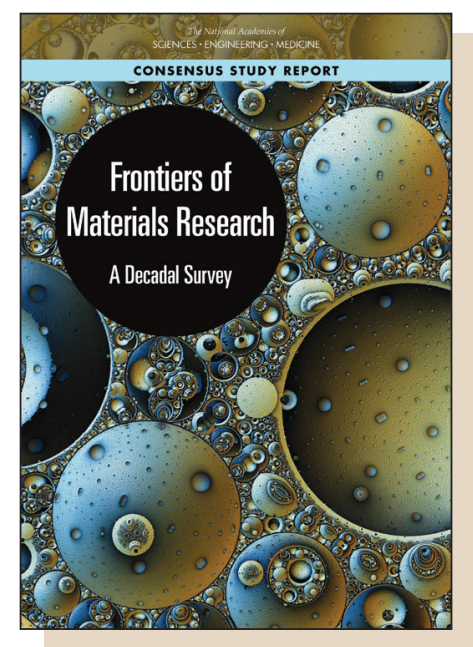

A Decadal Survey (doi:10.17226/25244), reviews developments in materials science over the last decade, examines a range of future opportunities in materials research, and makes recommendations to update government policy to better support this research into the coming decade.

Released in February 2019, the consensus report is the fourth in a series of studies on materials research by the Academies over the last four decades. The previous studies have been used to help inform and drive materials-related research and policy initiatives: Materials Science and Engineering for the 1990s: Maintaining Competitiveness in the Age of Materials (doi:10.17226/758), Materials Research to Meet 21st-Century Defense Needs (doi:10.17226/10631), and Condensed-Matter and Materials Physics: The Science of the World Around Us (doi:10.17226/11967).

The report is comprehensive, and Alan Hurd, executive advisor at Los Alamos National Laboratory and a member of the Academies Board on Physics and Astronomy, says it is "refreshingly organized by materials type, which took some courage given the remit to review a decade of advances." Containing a summary and five chapters, the report explores developments, progress, and achievements across materials science over the last decade (chapters 1-2); opportunities in materials research for the next decade (chapter 3); tools, methods, infrastructure, and facilities that enable research in materials (chapter 4); and an assessment on national competitiveness that includes case studies of a range of materials-enabled technologies (chapter 5). The findings and recommendations of the report, detailed in Table I, will be used to "inform science policymakers on appropriations, chief technology officers on investments, and department heads on new hires," Hurd says.
The summary of the report elucidates many of the important points:

\begin{abstract}
A remarkable number of paradigmchanging advances have been made in materials research over the past decade, and the pace of discovery is accelerating. Moreover, the tools that support that research-including capabilities for materials characterization, synthesis and processing, and computational modeling-have advanced considerably, enabling previously unachievable insights. The science and engineering are exciting, the prospects for creating and controlling new materials are high, and the pathway to important applications is very encouraging. This positive view is tempered somewhat by the prospect that many of the advances that can now be foreseen require resources that are by no means guaranteed, and by the reality that international competition, particularly from China and from Asia more generally, is threatening U.S. leadership in materials research.
\end{abstract}

Four members of the decadal study committee-Steven Zinkle, John Sarrao, Samuel Stupp, and Kevin Hemker - weigh in on the importance and timing, as well as the findings and recommendations, of the latest decadal survey. "The field of materials science is highly dynamic and continuously evolving," says Zinkle, UT/ ORNL Governor's Chair at The University of Tennessee and a member of the Academies National Materials and Manufacturing Board. "Therefore, it is valuable to have a periodic assessment of the major trends." But Zinkle also cautions that these studies "are not intended to predict potential future specific breakthroughs," and the report itself points out some significant areas, such as twodimensional materials (i.e., graphene) and the evolution of additive manufacturing, that were not foreseen by the previous 


\section{Table I. Key Findings and Recommendations from the Decadal Study}

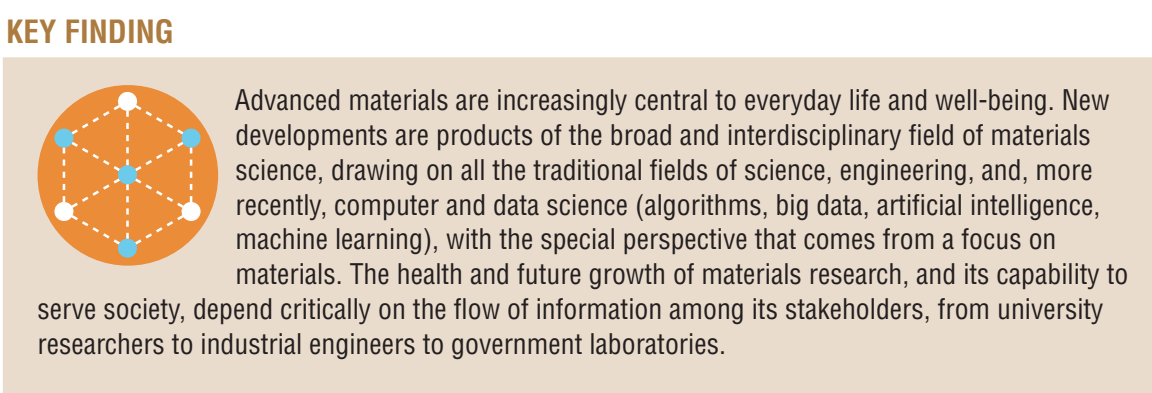

RECOMIMENDATION

*Government agencies, led by OSTP, should work with high priority to foster communication among materials research stakeholders through the support of interdisciplinary research and the development of modalities for freer flowing interactions among universities, private enterprise (including startup ventures), and national laboratories.

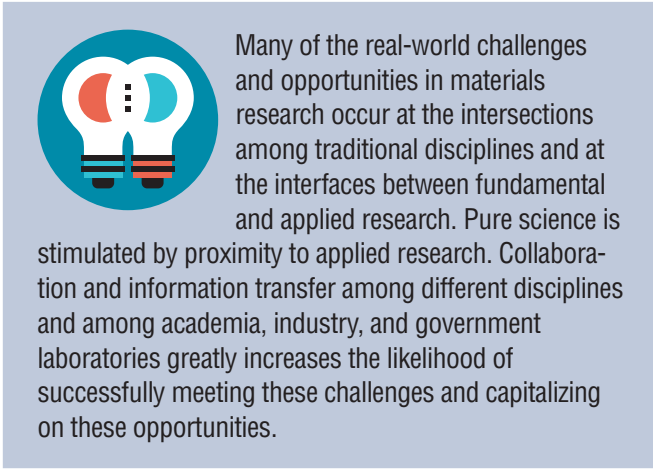

${ }^{*}$ Government agencies, led by OSTP, should work with high priority to foster communication among materials research stakeholders through the support of interdisciplinary research and the development of modalities for freer flowing interactions among universities, private enterprise (including startup ventures), and national laboratories.

OSTP should provide leadership in the development of awards that enable diverse funding agencies to work together when needed to facilitate collaboration among university and industry researchers.

NSF should develop a new type of center that will enable, and stimulate, students, faculty, and industrial scientists and engineers to work side by side. Such a Discovery to Translation Materials Research Center would create a unique learning and research environment. The effort should be supported by several NSF directorates and should continue for a minimum of a decade.

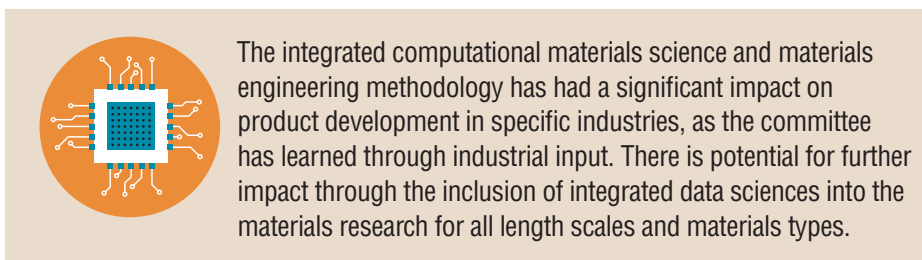

All government agencies funding materials research should encourage the use, when appropriate, of computational methods, data analytics, machine learning, and deep learning in the research they fund. They should also encourage universities to provide students of science and engineering exposure to these new methods by 2022 .

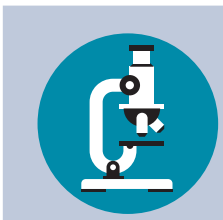

Basic research in fundamental science directions, meaning work that neither anticipates nor seeks a specific outcome, is the deep well that both satisfies the need to understand the universe and feeds the technological advances that drive the modern world. It lays the groundwork for future advances in materials science as in other fields of science and technology. Discoveries without immediate obvious application often represent great technical challenges for further development (e.g., high- $\mathrm{T}_{\mathrm{c}}$ superconductivity, carbon nanotubes) but can also lead to very important advances, often years in the future.

It is critically important that fundamental research remains a central component of the funding portfolio of government agencies that support materials research. Paradigmchanging advances often come from unexpected lines of work.

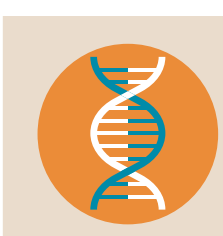
has resulted in numerous successful applications that have reduced the development time, with corresponding cost savings.
The Materials Genome Initiative, and the earlier Integrated Computational Materials Engineering approach, recognized the potential of integrating and coordinating computational methods, informatics, materials characterization, and synthesis and processing methods to accelerate the discovery and deployment of designer materials in
products. The translation of these methodologies to specific industries
Research into metals, alloys, and ceramics continues to provide fundamental understanding of atomic-scale processes that govern synthesis-microstructure-property relations in many classes of materials. Armed with this understanding and state-of-the-art synthesis, characterization, and computational tools, novel alloys and micro/nanostructures with extraordinary properties are being realized. Traditional areas of materials research can have surprising new developments, for example, in multicomponent, high-entropy alloys and inorganic glasses.
The US government, with NSF, DoD, and DOE coordinating, should support the quest to develop new computational and advanced data-analytic methods, invent new experimental tools to prove the properties of materials, and design novel synthesis and processing methods. The effort should be accelerated from current levels through judicious agency investments and continue over the next decade in order to sustain US competitiveness.

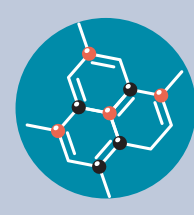

*Importance of intergovernmental and broader materials stakeholder communication.

Federal funding agencies (NSF, $\mathrm{DOE}, \mathrm{DoD}$ ) should maintain robust programs to support, and in some cases expand, fundamental research in long-established areas such as metals, alloys, and ceramics. 


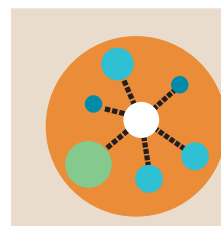

Quantum materials science and engineering, which can include superconductors, semiconductors, magnets, and two-dimensional and topological materials, represents a vibrant area of fundamental research. New understanding and advances in materials science hold the promise of enabling transformational future applications in computing, data storage, communications, sensing, and other emerging areas of technology. This includes new computing directions outside Moore's Law, such as quantum computing and neuromorphic computing, critical for low-energy alternatives to traditional processors. Two of NSF's " 10 big ideas" specifically identify support of quantum materials (see The Quantum Leap: Leading the Next Quantum Revolution and Midscale Research Infrastructure).
Significant investments by, and partnerships among, NSF DOE, NIST, DoD, and IARPA will accelerate progress in quantum materials science and engineering, which is crucial to the future economy and homeland security. US agencies with a stake in advanced computing, under the possible leadership of DOE's Office of Science and NNSA laboratories and the DoD research laboratories (ARL, NRL, AFRL), should support new initiatives to study the basic materials science of new computing paradigms during the next decade. To remain internationally competitive, the US materials research community must continue to grow and expand in these areas.

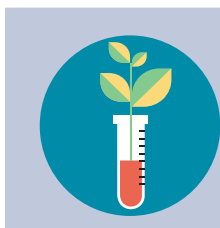

Materials science and technology has an enormous impact on the quality and sustainability of Earth's environment across the entire spectrum of materials types. This is another important opportunity for university, national laboratory, and industry cooperation.
Research in numerous directions that improve sustainable manufacturing of materials-including choices of feedstocks, energy efficiency, and recyclability-is urgently needed. NSF, DOE, and other agencies should develop creative approaches for funding materials research toward sustainability goals.

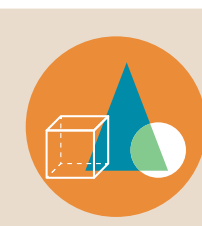

Progress in three-dimensional

characterization, computational materials science, and advanced manufacturing and processing have enabled an increasing digitization across disciplines of materials research and has, in some cases,

dramatically accelerated and compressed the time from discovery to inclusion in new products.

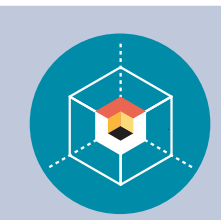

Infrastructure at all levels, from midscale instrumentation for materials characterization, synthesis, and processing with purchase costs of $\$ 4$ million to $\$ 100$ million in universities and national laboratories to large-scale research centers like synchrotron light sources, free electron lasers, neutron scattering sources, high field magnets, and superconductors, is essential for the health of the US materials science enterprise. Midscale infrastructure, in particular, has been sorely neglected in recent years, and the cost of maintenance and dedicated technical staff has increased enormously.

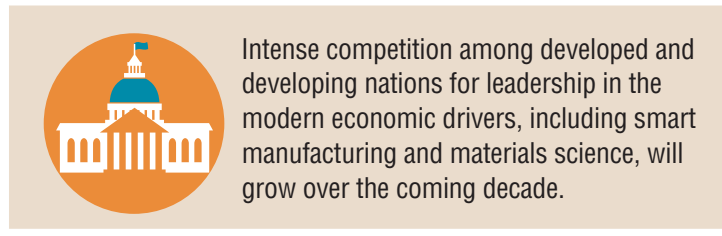

AFRL Air Force Research Laboratory

ARL Army Research Laboratory

DoD Department of Defense

DOE Department of Energy

IARPA Intelligence Advanced Research Projects Activity
Federal agencies (including NSF and DOE) with missions aligned with the advancement of additive manufacturing and other modes of digitally controlled manufacturing should, by 2020, expand investments in materials research for automated materials manufacturing. The increased investments should be across the multiple disciplines that support automated materials synthesis and manufacturing. These range from the most fundamental research to product realization, including experimental and modeling capabilities enabled by advances in computing, to achieve the aim that by 2030, the United States is the leader in the field.

All US government agencies with interests in materials research should implement a national strategy to ensure that university research groups and national laboratories have local access to develop, and continuing support for use of, state-of-the-art midscale instruments and laboratory infrastructure essential for the advancement of materials research. This infrastructure includes materials growth and synthesis facilities, helium liquefiers and recovery systems, cryogen-free cooling systems, and advanced measurement instruments. The agencies should also continue support of large facilities such as those at Oak Ridge National Laboratory, Lawrence Berkeley National Laboratory, Argonne National Laboratory, SLAC National Accelerator Laboratory, National Synchrotron Light Source II (Brookhaven National Laboratory), and National Institute of Standards and Technology and engage and invest in long-range planning for upgrades and replacements for existing facilities.

The US government, with input from all agencies supporting materials research, should take coordinated steps, beginning in 2020 , to fully assess the threat of increased worldwide competition to its leadership in materials science and in advanced and smart manufacturing. The assessment program, which should be established on a permanent basis, should also define a strategy by 2022 to combat this threat.

NIST National Institute of Standards and Technology

NNSA National Nuclear Security Administration

NRL Naval Research Laboratory

NSF National Science Foundation

OSTP White House Office of Science and Technology Policy 
decadal study. According to Zinkle, while the report cannot predict the future, it should be used to "provide a broader indication of emerging trends and opportunities as well as assessments of major accomplishments."

Sarrao, deputy director for science, technology, and engineering at Los Alamos National Laboratory, agrees that the report "informs both researchers and funding agencies on hot topics." But Sarrao also says that while many of the specific areas of research have changed significantly in the last decade, some constants, such as the continued "importance of integration among synthesis, characterization, and modeling in materials research," were also emphasized. This is important, according to Sarrao, because it provides a basis and motivation for what he calls "center-like funding modalities," which bring researchers and experts together to collaborate.

The need to foster communication within the materials research communityincluding national laboratories and government agencies, universities, and the private sector - is identified within the report as a "high priority." The report further acknowledges that materials science is highly interdisciplinary, posits that fundamental science is "stimulated by proximity to applied research," and proposes that the challenges facing materials researchers are best solved with collaboration. In its recommendations, the report tasks the White House Office of Science and Technology Policy (OSTP) with leading the government effort to develop "modalities for freer flowing interactions" among materials research stakeholders, and providing "leadership in the development of awards that enable diverse funding agencies to work together when needed to facilitate collaboration among university and industry researchers." In addition, the report recommends the development of a new type of center, which it calls a "Discovery to Translation Materials Research Center" that would both enable and stimulate collaboration between academics, industrial scientists and engineers, and federal scientists.

Stupp, Board of Trustees Professor of Materials Science \& Engineering, Chemistry, Medicine, \& Biomedical Engineering at Northwestern University, calls out the importance of "the continued efforts by federal agencies, particularly DOE, in developing further the crucial national facilities used currently to characterize materials." Stupp identifies this as one of the most significant factors in the progress of materials science over the last decade, and acknowledges that it will continue to be key to unlocking materials advancements well into the future. "The remarkable improvements in our experimental capabilities have led to a dependence on ever more sophisticated equipment whose cost grows with complexity," adds Hemker, professor and Alonzo G. Decker Chair in Mechanical Engineering at Johns Hopkins University. According to Hemker, the costs associated with conducting cutting-edge research will rise because of the need for "significant investments in infrastructure [that] will be critical to the continued advancement of materials research."

The report backs up the conclusions of Stupp and Hemker and calls for the implementation of a national strategy to ensure investment in, and access to, laboratory infrastructure from midscale instruments all the way to large facilities. The need to update midscale infrastructure, much of which is currently housed at universities, was specifically mentioned in the report as an area that needs immediate attention. The report notes that, in general, "the federal agencies, private foundations, and industry that fund most university research do not provide funding for the instrumentation that is needed to carry out that work." In effect, this means that universities are increasingly responsible for funding midscale research instruments, which is often done as part of a package to attract young talent. The downside of this practice, according to the report, is that it lacks sustained funding to upgrade or support the instrumentation over time. In addition, the report highlights the need for long-range planning for both upgrades and new large-scale facilities.

While the report surveys the breadth of materials research, certain fields of elevated importance are identified because of their effect across a broad range of technologies. One such field is the application of computational methods (data science, machine learning, informatics, and artificial intelligence) to materials research. "I do not think that we can underestimate the transformation effect that increased computational power is having on all fields of science and engineering, and materials science and engineering is no exception," Hemker says. "The industrial surveys we [the decadal study committee] received indicated a tremendous buy-in for Integrated Computation Materials Engineering approaches and the Materials Genome Initiative, and framed the important interplay between computation, experiments, and data."

The evidence of the usefulness of computational methods in materials research is undeniable, and the report recommends that government agencies, with NSF, DOE, and Department of Defense (DoD) coordinating, should invest funds and develop new computational methods for materials research. It also recommends that all government agencies that fund materials research should encourage the use of these computational methods where applicable, and that universities should provide students in science-based fields with exposure to these new methods by 2022. "We are ushering in a digital revolution in materials research that will benefit both fundamental discovery and accelerate materials development and insertion into commercial products," Hemker says. "The next wave of this revolution will involve the use of machine learning and artificial intelligence to facilitate quantification, sorting, filtering, and interpretation of the digital data that we are producing."

Developments in digital manufacturing also show up in the report's key findings. Driven by advances in computational materials science and three-dimensional characterization, additive manufacturing and other digitally controlled manufacturing methods have rapidly advanced. According to the report, this has enabled new materials to progress from discovery to use on an accelerated timeline. Increased investment from NSF, DOE, and other federal agencies that support research on digitally controlled manufacturing is recommended in an effort to achieve world leadership in this field by 2030 . 
Materials for quantum information science (QIS) also emerge from the report as a focus issue. QIS already has the attention of scientists and policymakers, as evidenced by the recent passage of the National Quantum Initiative Act (reported in the March issue of MRS Bulletin). According to the report, the development of QIS technologies will have a significant effect on the economy and national security. The report recommends partnerships between, and investments from, NSF, DOE, the National Institutes of Science and Technology, DoD, and the Intelligence Advanced Research Projects Activity to accelerate progress in materials research for QIS.

The report also calls out the impact of materials science and technology on sustainability and the environment. Presented as an opportunity for materials researchers to both collaborate and have a significant impact, the report says research to improve sustainable manufacturing of materials is "urgently needed" and recommends that NSF, DOE, and other agencies develop "creative approaches for funding materials research toward sustainability goals." Hurd points out the importance of sustainability being included among the "key" recommendations of the report, saying, "sustainability in resource consumption will haunt society until we 'close the loop,' and eventually the availability of research-quality materials will be affected if we don't work to address this issue."

One additional finding from the report, which may be easy to overlook beside the exciting new advances in materials research and the need to address sustainability, is the importance of continued focus on fundamental research. "This is an area where MRS has been particularly active in advocating for strong continued support across the agencies for fundamental materials research," Dozier says. The report asserts that basic research satisfies the human need to better understand the world while also laying the groundwork for future discoveries. In materials, although areas that the report terms "traditional—such as metals, alloys, and ceramics - have long been studied, fundamental research is still returning new understanding of synthesis-microstructure-property relationships. The report recommends that NSF, DOE, and DoD all maintain "robust" funding for fundamental research across these areas and even encourages expanding research efforts in some cases. The related general recommendation for support of basic research says, "It is critically important that fundamental research remains a central component of the funding portfolio of government agencies that support materials research. Paradigm-changing advances often come from unexpected lines of work."

The final chapter of the report examines how materials research in the United States compares internationally. "From a domestic US perspective, international competitiveness is a challenge," Sarrao says. He points out that the report details the significant expansion of both the quality and scale of materials research efforts across a number of different countries. China's increase in R\&D spending is specifically mentioned within the report. And Asia is more generally called out as a region of rising importance across research areas, including materials science. In an attempt to mitigate the risk of falling behind, the report recommends that the US government solicit input from all agencies that support materials research to assess what it terms the "threat of increased worldwide competition to its leadership in materials science." The report recommends beginning the assessment in 2020 and defining a strategy to combat the threat by 2022 .

Sarrao says that international competition in materials research "creates both an opportunity for enhanced global collaboration and cooperation, and a reminder to US policymakers that we need to remain committed to a robust domestic materials research enterprise, or we risk falling behind in this economically important field." Zinkle says that one of the biggest challenges that could slow the pace or stall areas of materials research in the United States is "uncertainties in research planning due to budgetary oscillations." And Stupp expresses his hope that one outcome of the report will be a reminder to the US government that "the critical role of science and technological innovation is precisely what has made this country the most important economy in the world."

The frontiers of materials research are clearly expanding, and according to the report "will have a tremendous effect on our understanding, and significant impact on our daily lives, globally." The findings and recommendations laid out here will undoubtedly impact materials research and policy in the United States over the next decade and beyond. But, at least one materials expert thinks this may be the final review of materials research covering a full decade. "It may be impossible to perform such a comprehensive review 10 years from now because materials research is advancing exponentially fast," Hurd says. So what does this mean for the materials community? According to Hemker, it means that "there has never been a better time to be a materials researcher."

Jennifer A. Nekuda Malik

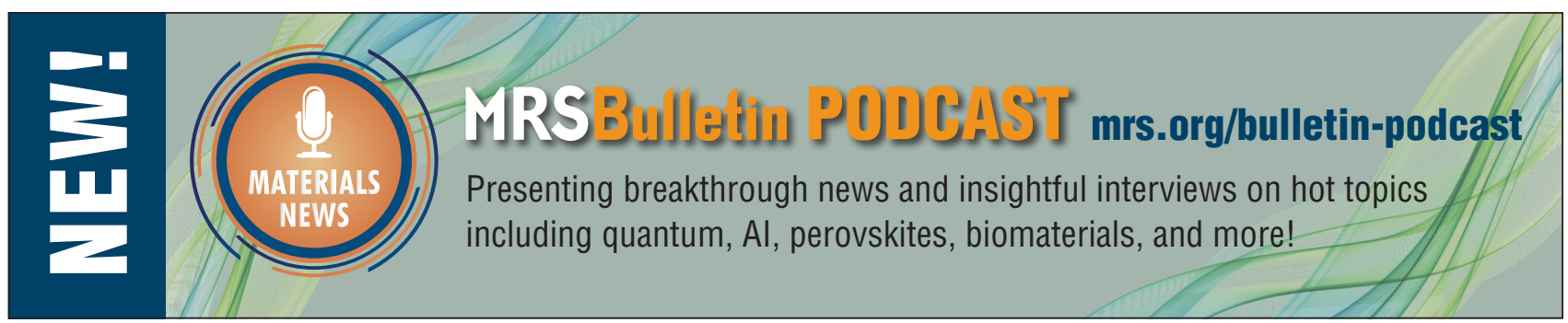




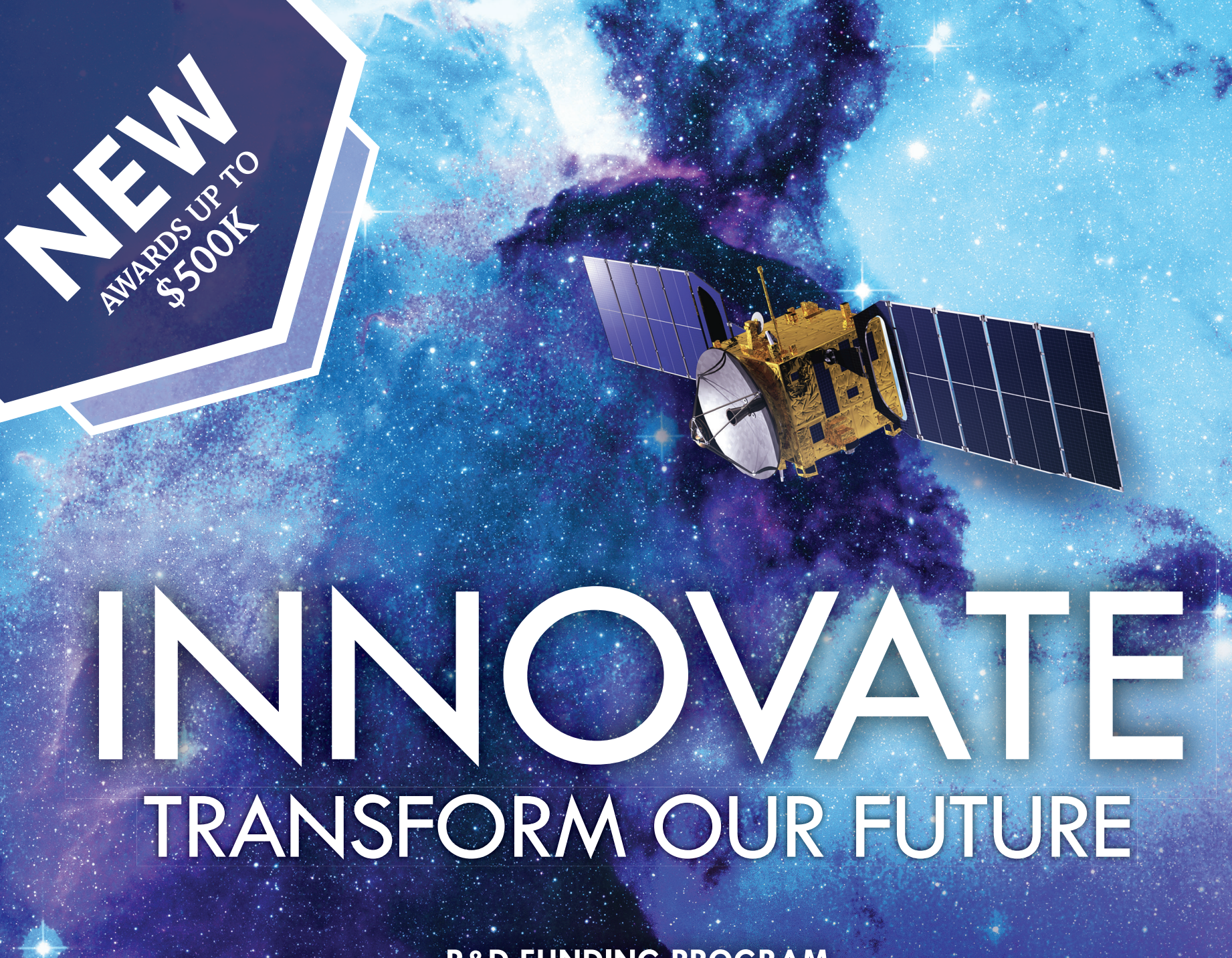

\section{R\&D FUNDING PROGRAM}

The National Reconnaissance Office Director's Innovation Initiative (DII)

Program funds cutting-edge scientific research in a high-risk, high-payoff environment to discover innovative concepts and creative ideas that transform overhead intelligence capabilities and systems for future national security intelligence needs. The program seeks the brightest minds and breakthrough technologies from industry, academia, national laboratories, and U.S. government agencies.

Visit the website for Broad Agency Announcement and Government Sources Sought Announcement requirements.

\subsubsection{9}
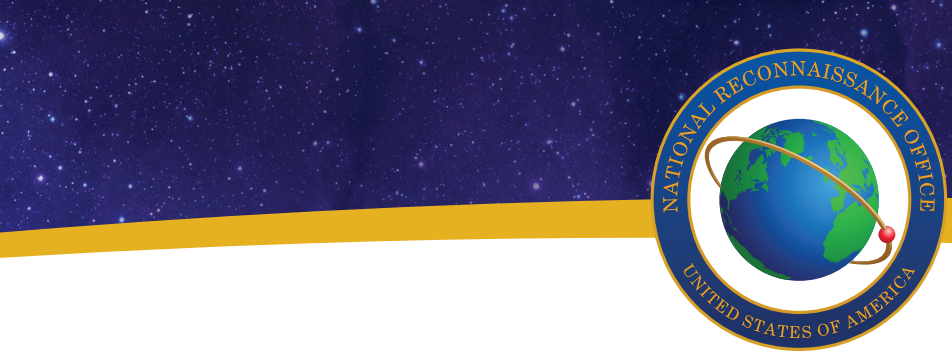

www.nro.gov/About-the-NRO/Business-Opportunities 